
\title{
BReserch S Suare \\ Impact of Event Scale-6 (IES-6) for U.S. Adults Who Experienced the COVID-19 Pandemic
}

\author{
Jiin Jeong \\ Yonsei University \\ Ah-Ram Kim \\ Yonsei University \\ Claudia Hilton \\ University of Texas Medical Branch \\ Ickpyo Hong ( $\square$ ihong@yonsei.ac.kr) \\ Yonsei University
}

\section{Research Article}

Keywords: COVID-19, coronavirus, impact of event scale-6, post-traumatic stress disorder, Rasch analysis, psychometrics

Posted Date: December 20th, 2021

DOI: https://doi.org/10.21203/rs.3.rs-1130260/v1

License: (c) (1) This work is licensed under a Creative Commons Attribution 4.0 International License. Read Full License 


\section{Abstract}

Background: Post-traumatic stress disorder (PTSD) is a mental illness caused by traumatic events, such as the COVID-19 pandemic. Accurate diagnosis of this disorder is critical to establish effective intervention approaches. The Impact of Event Scale-6 (IES-6) is widely used for PTSD screening, but there has been no research on its psychometric properties with individuals who experienced the COVID-19 pandemic.

Methods: A random sample of 600 participants were randomly selected from a COVID-19 survey database $(n=6,391)$. Rasch analysis was conducted to examine item fit, rating scale structure, construct validity, differential item functioning (DIF), and precision of the IES-6.

Results: The principal component analysis of Rasch residuals (54.1\% of the raw variance explained) and the average of residual correlations (average $r=.19$ ) supported the unidimensionality structure in the IES6. The rating scale was suitable, and the item difficulty hierarchy was logical. The item fit and the DIF contrast were acceptable, except for item 5 . The IES-6's person reliability was .76, which was also an acceptable level.

Conclusions: This study showed that the IES-6 has acceptable item-level psychometrics for screening PTSD in adults in the United States for individuals who have experienced the COVID-19 pandemic. The findings suggested that the IES- 6 would be useful for the rapid identification of PTSD and allow clinicians to quickly provide interventions for people with the disorder and their families.

\section{Introduction}

Coronavirus disease (COVID-19) was first identified in Wuhan, China, and the World Health Organization declared the epidemic on March 11, 2020 due to increased human-to-human transmission [1]. COVID-19 has a very high infection rate and a relatively high mortality rate $(3.6 \%$ and $1.5 \%$ in and outside of China, respectively [2]). Because the disease affects people of all age groups, widespread infection prevention policies, such as "social distancing" and "shelter in place" have been adopted [3, 4].

Pandemic-related issues, such as social distancing and isolation, have amplified the fear of stigma in some cases [5]. Such isolation can have a detrimental effect on an individual's physical and mental health [6]. It can also cause health problems other than COVID-19 infections, such as psychological pain and fear [7], and mental health problems (e.g., depression, stress, panic, distress, etc.) can lead to suicide accidents, suicide attempts, and actual suicide occurrences [8].

COVID-19 affects both physical and mental health [9]. Disasters such as the COVID-19 pandemic often lead to the diagnosis of mental disorders, such as post-traumatic stress disorder (PTSD), adjustment disorders, anxiety disorders, non-specific somatic symptoms, and substance abuse $[10,11]$. Researchers have designated PTSD as a representative diagnosis of mental illness after a disaster [10]. 
A prior study showed that the difference between positive and negative emotions increased after the COVID-19 pandemic by the presence of anxiety, depression, and anger, while positive emotions and life satisfaction decreased [12]. In addition, evidence has shown that cases of PTSD were common after the COVID-19 outbreak [13]. PTSD symptoms are associated with long-lasting reduction in the ability to perform daily life activities [14] and reduction in quality of life [15], which are serious consequences for survivors and their families [16]. Therefore, clinical detection of PTSD symptoms is essential to be aware of potential consequences in well-being and quality of life.

Identifying the psychological problems that need to be addressed to help protect well-being and psychological health under COVID-19 is of paramount importance [17]. The accurate diagnosis of psychiatric syndromes associated with COVID-19 is an important first step toward best practice. It guides clinicians to choose the most appropriate and effective treatment and helps to be aware of potential prognoses [18]. A valid and reliable brief screening instrument for PTSD can be a valuable first stage in this process.

The Impact of Event Scale (IES)-6 consists of six items (total point range: 0-24) to quantify PTSD symptoms [19]. The IES-6 was developed based on the widely used Impact of Event Scale-Revised (IESR). The validity and reliability of the IES-6 have been well evaluated [20]. However, information on the psychometric properties of the scale has yet been examined. Therefore, the objective of this study is to assess the internal consistency, criterion validity, and external construction validity of the IES-6 through Rasch analysis.

\section{Methods}

\section{Participants}

This study utilized the survey data from "Knowledge, attitudes, and practices related to COVID-19 in the U.S.," registered in the Inter-university Consortium for Political and Social Research (ICPSR) [21]. This national survey sought to assess the state of COVID-19-related knowledge, beliefs, mental health, substance use changes, and behaviors among a sample of adults aged 18 years or older currently residing in the United States. The survey was administered online from March 20-30, 2020. Depression and anxiety were assessed using the Patient Health Questionnaire-4, stress was assessed using the Impact of Event Scale-6, and pessimism and changes in tobacco and alcohol use were assessed by the responses to the questionnaire. A total of 6,391 respondents met the eligibility requirements [22]. The study data is a publically avaliable open data set, and all methods were carried out in accordance with the local university's guidelines and regulations for use of Human data.

\section{Outcome Measures}

To assess the subjective stress of COVID-19, the items of the Impact of Event Scale-6 (IES-6) were adapted (Supplementary Table 1, Additional file 1). The test items consisted of a 4-point Likert scale 
ranging from 0 to $3(0=$ not at all; $1=$ several days; $2=$ more than half the days; $3=$ nearly every day). The summed score ranged from 0 to 18 , with higher scores indicating greater PTSD symptoms.

The IES-6 [23] is a 6-item short version of the Impact of Event Scale-Revised (IES-R) [24] that measures the principal components of PTSD [18]. The IES- 6 demonstrated good sensitivity $(r=.88)$ and specificity $(r=.85)$ with a standard PTSD semi-structured interview conducted by physicians $[20,25]$.

\section{Data analysis}

Of the total subjects $(n=6,391), 600$ random samples (approximately 10\%) were analyzed. Descriptive statistics were used to examine participants' demographic characteristics. Unidimensionality was examined using principal component analysis (PCA) of Rasch residuals. In addition, once the instrument revealed a single dominant measurement structure, we conducted item-level analysis using the Rasch model, including rating scale analysis, item fit statistics, precision, differential item functioning (DIF), and construct validity. Statistical analyses were performed using SAS v. 9.4 and Rasch analysis was performed using Winsteps v. 4.7.1.

\section{Unidimensionality}

\section{Principle component analysis (PCA) of Rasch residuals}

Principal component analysis (PCA) of the residuals was used to examine the unidimensionality assumption in the test items [26]. Unlike conventional factor analysis, PCA of Rasch residuals is performed after excluding the target configuration, and secondary dimensions are detected.

Unidimensionality assumes that the eigenvalue for the first contrast is less than 2.0 or that the variance ratio explained by the measurement is greater than $20 \%$ [27].

\section{Local independence}

Local independence means that when the structure level is controlled, the response to the item is not related to another item. To identify local independence, the residual correlation matrix was examined [28]. An average item residual correlation exceeding .20 was interpreted as indicating dependency [29].

\section{Rasch analysis}

\section{Rating Scale Analysis and Item Fit Statistics}

The rating scale model was applied to the six items of the IES-6. To examine the fit of the data to the Rasch model, a rating scale analysis was used. We determined the extent to which the empirically obtained data matched the predictions of the model.

Rating scale analysis criteria. 1) at least ten observations in each rating scale, 2) monotonically advanced average measure in rating scale categories, and 3 ) outfit mean squares (MnSq) less than 2.0 for rating scale categories [30]. 
Item fit. We used the mean square residual (MnSq) and standardized mean square residual (Zstd) to examine item fit. MnSq values between .6 and 1.4 and Zstd values between -2.0 and 2.0 indicate acceptable fit [31]. It is agreed that up to $5 \%$ of the sample could demonstrate misfit without being a serious threat to validity [32]. The values provided by this model are expressed in the logit scale, which is a logistic transformation of the observed scores with a mean of 0 and a standard deviation of 1 . According to construct theory, suitable items can measure intended unidimensionality and Rasch analysis is a powerful tool for evaluating construct validity [33]. The conditional maximal likelihood estimator (CMLE) was used for the parameter consistency [34].

\section{Validity}

Construct validity. The sequence of reasonable or conceptual item difficulties for the assessment item is interpreted as construct validity [33]. In the Rasch model, the difficulty of the evaluation items and the IES6 score are located in the same linear continuum (logit), and the matching between the evaluation items and the human measurement is presented by the Wright map. We analyzed whether the sequence of difficulty layers of the estimated evaluation items in the Rasch model matched the logical progression from the easiest to the most challenging. Furthermore, we examined whether ceiling and floor effects were at least $5 \%$ of the samples in measurements with the maximum and minimum criteria [35].

Convergent validity. To secure convergent validity, the person measure was correlated with the PHQ-4, which assesses anxiety and depression symptoms. Spearman correlation analysis was used to examine the correlation between PHQ-4 and the IES-6 scores.

\section{Differential Item Functioning (DIF)}

In Rasch and item response theory models, the probability of item responses should be a function of the basic characteristic level of people [36]. We conducted a DIF to examine the linear invariant estimation of item difficulty parameters based on the Rasch model [37]. If different group members have the same characteristic level but different response probabilities, the entry represents differential item functioning (DIF). We used the Rasch-Welch t test to compute the size of the DIF [38]. The following are the effect criteria for DIF: (a) a moderate to large DIF (greater than .64 logits in the DIF contrast, thus indicating the difference in item difficulties between the two comparison groups) and (b) a slight to moderate DIF (greater than .43 in the DIF contrast). The significance of DIF contrast was determined at an alpha value of .05 with a two-sided Rasch-Welch ttest [38].

\section{Precision}

Personal reliability reflects the degree of impact of measurement scores on measurement errors [39]. In Rasch analysis, every participant is given a Rasch score with an individual person reliability. Personal reliability used the sum score of IES-6 for group comparisons: a score of .7 or higher was considered suitable and .9 or higher was suitable for comparing individual reliability [40]. A person separation index of 2.00 indicates acceptable levels of separation, where a value of 3.00 represents a good separation 
level. We calculated Cronbach's alpha to examine the internal consistency of the instrument. A value of .70 is considered acceptable, .80 is good, and .90 is considered excellent [41].

\section{Results}

\section{Demographic characteristics}

The demographic characteristics of participants are displayed in Table 1. The sample comprised 600 participants from the COVID-19 online survey. Females accounted for $59 \%$ of the participants $(n=354)$. The highest percentage of respondents were in their $50 \mathrm{~s}$, followed by those in their $60 \mathrm{~s}, 40 \mathrm{~s}$, and $30 \mathrm{~s}$. A majority $62.7 \%(n=376)$ were employed. A total of $29.7 \%(n=178)$ of participants had children under the age of 18 and $70.3 \%(n=422)$ had no children. There were no significant differences between the total and sample groups.

Table 1 Demographic characteristics of participants 


\begin{tabular}{|c|c|}
\hline Variable & Sample, $n(\%)$ \\
\hline \multicolumn{2}{|l|}{ Sex } \\
\hline Female & $354(59.0)$ \\
\hline Male & $244(40.7)$ \\
\hline Other/No disclosure & $2(.3)$ \\
\hline \multicolumn{2}{|l|}{ Age group (years) } \\
\hline $18-29$ & $59(9.8)$ \\
\hline $30-39$ & $86(14.3)$ \\
\hline $40-49$ & $108(18.0)$ \\
\hline $50-59$ & $187(31.2)$ \\
\hline $60-69$ & $121(20.2)$ \\
\hline$\geq 70-79$ & $39(6.5)$ \\
\hline \multicolumn{2}{|l|}{ Residence type } \\
\hline Rural & $184(30.7)$ \\
\hline Suburban & 313 (52.2) \\
\hline Urban & 103 (17.2) \\
\hline \multicolumn{2}{|l|}{ Employment status } \\
\hline Work & $376(62.7)$ \\
\hline Unemployed & $80(13.3)$ \\
\hline Student/Retired/Unpaid work (e.g., homemaker, eldercare, childcare) & $144(24.0)$ \\
\hline \multicolumn{2}{|l|}{ Work in healthcare/clinical setting } \\
\hline Yes & $100(16.7)$ \\
\hline No & $500(83.3)$ \\
\hline \multicolumn{2}{|l|}{ Has children under 18} \\
\hline Yes & $178(29.7)$ \\
\hline No & $422(70.3)$ \\
\hline \multicolumn{2}{|l|}{ Educational attainment } \\
\hline High school or below & $94(15.7)$ \\
\hline Some college & $205(34.2)$ \\
\hline
\end{tabular}




\section{Rasch analysis}

\section{Unidimentionality}

The Rasch model explained $54.1 \%$ of the raw variance in PCA of Rasch residuals. The eigenvalues of the first, second and third contrast were $1.43(11.0 \%), 1.32(10.1 \%)$ and $1.09(8.4 \%)$, respectively. The average of residual correlation was $.19(<.20)$.

\section{Rating scale analysis}

The IES-6 for COVID-19 utilizes a 4-point Likert scale, and the results of checking the suitability of the categories are listed in Supplementary Table 2, Additional file1. According to the rating scale analysis, the four response categories were all selected more than ten times, had a good fit (outfit MnSq ranges .821.08), and average measures and Andrich thresholds (step calibration) were increased sequentially.

\section{Fit statistic}

The infit and outfit of the Rasch model were checked to determine the goodness of fit of the items of the IES-6 for COVID-19. Most of the items were found to be suitable, but in item 5 ("I tried not to think about the coronavirus"; $\mathrm{MnSq}=1.63, \mathrm{Zstd}=9.05), \mathrm{MnSq}$ was found to be greater than 1.4 and Zstd was greater than 2.0, which made it a misfitting item. However, the contents of item 5, indicating avoidance of stresscausing factors, have not been deleted as they are the main clinical aspects of PTSD [42]. Table 2 presents the item-fit statistics.

The percentage of misfitting persons was $7.33 \%(n=44)$, but the item parameter was invariant even if the misfit individuals were removed.

\section{Table 2}

Item fit, Item difficulty hierarchy results 


\begin{tabular}{|lllllll|}
\hline Item number & Measure & Model & Infit & \multicolumn{3}{c|}{ Outfit } \\
\cline { 2 - 7 } & \begin{tabular}{l} 
(logits) \\
\cline { 5 - 7 }
\end{tabular} & SE & MnSq & Zstd & MnSq & Zstd \\
\hline 2 & -.53 & .06 & .76 & $-4.43^{\mathrm{b}}$ & .74 & $-4.25^{\mathrm{b}}$ \\
\hline 3 & -.83 & .06 & 1.12 & 2.00 & 1.20 & $2.66^{\mathrm{b}}$ \\
\hline 4 & -.31 & .06 & .79 & $-3.93^{\mathrm{b}}$ & .80 & $-3.33^{\mathrm{b}}$ \\
\hline 5 & .86 & .06 & .93 & -1.11 & .80 & $-2.90^{\mathrm{b}}$ \\
\hline 6 & .20 & .06 & $1.63^{\mathrm{a}}$ & $9.05^{\mathrm{b}}$ & $1.70^{\mathrm{a}}$ & $9.18^{\mathrm{b}}$ \\
\hline
\end{tabular}

Note. $\mathrm{MnSq}=$ mean square, $\mathrm{Zstd}=$ standardized mean square

${ }^{a}$ MnSq value less than .6 or more than 1.4 , Zstd value less than -2.0 or more than 2.0 .

\section{Validity}

Construct validity. The item difficulty hierarchy of the assessment items is presented in Table 2. Item 4 was the most difficult item, and Item 2 was the easiest. In addition, the difference between the item average and the person average was less than .5 logits. Left of Figure 1 indicated distribution of person ability, and right showed item difficulty. Person ability scores were distributed between -4 and 4 , and items were placed within this range. The number of people who received the maximum score was $47(7.8 \%)$ and the minimum score was $23(3.8 \%)$. The maximum was slightly greater than $5 \%$, but it can be considered to have no ceiling or floor effect (Figure 1).

Convergent validity. To assess convergent validity, we calculated the correlation with the PHQ-4 total score, the tool included in the same database. The PHQ-4 was used to evaluate anxiety and depression symptoms among people who experienced the COVID-19 pandemic. Spearman's correlation revealed a significant correlation between the two assessment tools $(r=.109, p=.007)$.

\section{DIF}

Following prior studies, the DIF contrast of age, sex, residence type, working status, having children under the age of 18, and healthcare or clinic of profession were identified as indicative of PTSD involvement $[43,44]$. The age group was divided into two groups based on the age of 50 years, based on a previous study by Karatzias et al. [43], which suggested that there was a significant difference in the number of people diagnosed with PTSD related to COVID-19. Item 5 showed significant DIF with or without children under 18 (DIF contrast $=0.47, p=.0003$; Table 3 ). There were no significant differences in the DIF among the other variables. 


\section{Table 3}

Differential item functioning across having children under the age of 18

\begin{tabular}{llllll}
\multirow{2}{*}{ Item number } & \multicolumn{5}{l}{ Having children under 18 years old (No vs. Yes) } \\
\cline { 2 - 6 } & DIF & Joint & \multicolumn{4}{l}{ Rasch-Welch } \\
\cline { 2 - 6 } & Contrast & SE & $t$ & $d f$ & Prob. \\
\hline 1 & -.26 & .13 & -2.04 & 270 & .04 \\
\hline 2 & -.17 & .13 & -1.33 & 267 & .19 \\
\hline 3 & .21 & .12 & 1.73 & 275 & .08 \\
\hline 4 & -.07 & .13 & -.56 & 289 & .58 \\
\hline 5 & $.47^{\text {a }}$ & .13 & 3.71 & 272 & $.00^{*}$ \\
\hline 6 & -.21 & .13 & -1.62 & 289 & .11
\end{tabular}

Note. Reference group = Having children under 18 years old, DIF = differential item functioning

${ }^{a}$ Absolute value of DIF contrast over .43

${ }^{*} p<.001$

\section{Precision}

The IES- 6 for COVID-19 had an acceptable person reliability of .76. In addition, the person separation index was 1.60, 2.47 person strata, which is an acceptable level. The Cronbach's a was .86.

\section{Discussion}

The IES-6 has been widely used as a tool for PTSD screening [23]. However, it was necessary to ensure that this tool was also suitable for the measurement of post-traumatic stress induced in the special situation of the COVID-19 pandemic. This study aimed to verify the validity of the items and rating scale categories of the IES-6 adjusted for COVID-19. The IES-6 showed validity in all items except item 5, and the response category was also appropriate.

Rasch analysis was used to validate the results. The eigenvalue, derived from the PCA of Rasch residuals, indicated that this tool satisfies the unidimensional assumption of Rasch analysis. In addition, local independence between items was identified by the average of the residual correlation. It means that the six items of the IES-6 explain one factor, which we refer to as "coronavirus PTSD." When these 
assumptions are satisfied, it can be seen that the Rasch model theory can be applied [26]. This process is different from confirming the validity using the 3 -factor model, as seen in another study [19]. The difference in traumatic experience, i.e., the bank robbery, seems to have had an effect.

The IES- 6 had a logical hierarchy of each item's disparity; item 4 was the most difficult and item 2 was the easiest. Owing to the strong transmission power of the coronavirus, it is possible that most of the participants chose a low score on item 2 ("I felt watchful or on-guard"). In this context, the respondents may have given high scores on item 4 ("I was aware that I still had a lot of feelings about the coronavirus, but I didn't deal with them.") because it is difficult to deal with the idea of the virus in a situation where personal and social COVID-19 prevention continues [4]. Figure 1 is a Wright map that visualizes the hierarchy of these items. In the map, item difficulty and person ability are similarly distributed. Through the distribution of person ability and the item difficulty, it was found that IES- 6 could measure the overall level. Matching between the two would reduce the likelihood of errors that could occur in personal measurement estimation [45].

Both ceiling and floor effects were acceptable and did not threaten the validity of the evaluation tool. In addition, the five items had goodness-of-fit and there was no DIF. However, item 5 ("I tried not to think about the coronavirus") was found to be a misfit item through fit statistics, and the estimated average was higher than those without children. These values were consistent with those of other studies, which show that people pay more attention to the virus when they have children [43]. This item is important because it represents an important clinical characteristic indicating the avoidance of the cause of stress, one of the major symptoms of PTSD screening [42]. In addition, if the item was deleted, person reliability was degraded and could not be removed.

When item 5 was not excluded, person reliability and person separation indexes indicated high precision in measuring coronavirus-related stress. Cronbach's alpha was also acceptable. The scores of the PHQ-4 and IES-6 included in the same questionnaire were statistically significant, and consistent with those of previous studies, which indicate that patients with PTSD had depressive and anxiety symptoms [20]. This correlation shows the convergent value of IES-6.

\section{Limitations}

The participants in this study consisted only of adults residing in U.S. who could access Facebook. These restrictions prevent adolescents and individuals from other cultures that would allow us to generalize whether the IES-6 is appropriate. In addition, it was not possible to measure the mental health of individuals with severe disabilities, who could not use Facebook. The COVID-19 pandemic is known to have had a greater impact on them and has a longer-term impact on their mental health [46]. Therefore, it is necessary to collect and analyze the results through a wider survey in the future. Second, due to the nature of the online survey, there is a possibility that answers will not always be accurate, so the ratio of misfit persons was slightly higher. However, it can be considered that misfit persons did not affect this result because there was no difference even when they were deleted. Finally, it is important to pay 
attention to the interpretation of the results because item 5 had a misfit but could not be deleted. After that, it is necessary to supplement and improve the questions so that PTSD can be selected more accurately.

\section{Conclusion}

Despite the misfitting of one item, the results of this study indicate that the IES- 6 is acceptable for PTSD screening in American adults, who Have experienced the COVID-19 pandemic. The IES-6 for COVID-19 showed acceptable psychometric properties in unidimensionality, rating scale, fit statistics, DIF, and precision. COVID-19-related PTSD is a disease that affects many aspects of an individual's daily life. Therefore, rapid screening and appropriate approaches are needed, and it is suggested that the use of the IES-6 can be useful in this process.

\section{Declarations}

\section{Ethics approval and consent to participate}

This research protocol has been reviewed and approved by the Institutional Review Board of Yonsei University Mirae Campus (1041849-202110-SB-164-01).

\section{Consent for publication}

Not applicable.

\section{Availability of data and materials}

The dataset supporting the conclusions of this article is available in the [Inter-university Consortium for Political and Social Research (ICPSR)] repository, [unique persistent identifier and hyperlink to dataset in https://www.openicpsr.org/openicpsr/project/120308/version/V1/view].

\section{Competing interests}

The authors declare that they have no competing interests.

\section{Funding}

This work was supported by the Ministry of Education of the Republic of Korea and the National Research Foundation of Korea (NRF-2021S1A3A2A02096338)

\section{Authors' contributions}

AK, JJ conceptualized and designed the study. JJ conducted statistical analysis. AK, JJ draft the preliminary manuscript. $\mathrm{IH}, \mathrm{CH}$ provided edits to the manuscript. All authors read and approved the final manuscript. 
Acknowledgements

We would like to thank Sang-Hun Nam for advice on statistical methods and Editage (www.editage.co.kr) for English language editing.

\section{Author's information}

1 Jiin Jeong, BS, OT, Integrative Course, Department of Occupational Therapy, Graduate School, Yonsei University, 135 Backun Hall, 1 Yonseidae-gil, Wonju-si, Gangwon-do, Republic of Korea.

${ }^{2}$ Ah-Ram Kim, MS, OT, Doctoral Student, Department of Occupational Therapy, Graduate School, Yonsei University, 135 Backun Hall, 1 Yonseidae-gil, Wonju-si, Gangwon-do, Republic of Korea.

${ }^{3}$ Claudia Hilton, Ph.D., OTR, MBA, FAOTA, Associate Professor, Department of Occupational Therapy, University of Texas Medical Branch, Galveston, Texas, USA.

Corresponding Author. ${ }^{4}$ Ickpyo Hong, Ph.D., OTR, Assistant Professor, Department of Occupational Therapy, College of Software and Digital Healthcare Convergence, Yonsei University, 135 Backun Hall, 1 Yonseidae-gil, Wonju-si, Gangwon-do, Republic of Korea.

\section{References}

1. World Health Organization. WHO director-general's opening remarks at the media briefing on COVID19. [Internet]; 2020. https://www.who.int/dg/speeches/detail/who-director-general-s-openingremarks-at-the-media-briefing-on-covid-19--11-march-2020. Accessed 11 March 2020.

2. Baud D, Qi X, Nielsen-Saines K, Musso D, Pomar L, Favre G. Real estimates of mortality following COVID-19 infection. Lancet Infect Dis. 2020;20(7):773. https://doi.org/10.1016/S14733099(20)30195-X.

3. Liu W, Zhang QI, Chen J, Xiang R, Song H, Shu S, et al. Detection of COVID-19 in children in early January 2020 in Wuhan, China. N Engl J Med. 2020;382(14):1370-1371. http://doi.org/10.1056/NEJMc2003717.

4. World Health Organization. Coronavirus disease 2019 (COVID-19): Situation Report. 72 [Internet]; 2020. https://apps.who.int/iris/handle/10665/331685. Accessed 1 April 2020.

5. Lin CY. Social reaction toward the 2019 novel coronavirus (COVID-19). Soc Health Behav. 2020;3(1):12.

6. Banerjee D, Rai M. Social isolation in Covid-19: the impact of loneliness. Int J Soc Psychiatry. 2020;66(6):525-527 http://doi.org/10.1177/0020764020922269.

7. Ahorsu DK, Lin CY, Imani V, Saffari M, Griffiths MD, Pakpour AH. The fear of COVID-19 scale: development and initial validation. Int J Ment Health Addiction. 2020:1-9. https://doi.org/10.1007/s11469-020-00270-8. 
8. Goyal K, Chauhan P, Chhikara K, Gupta P, Singh MP. Fear of COVID 2019: first suicidal case in India! Asian J Psychiatr. 2020;49:101989. http://doi.org/10.1016/j.ajp.2020.101989.

9. Lai J, Ma S, Wang Y, Cai Z, Hu J, Wei N, et al. Factors associated with mental health outcomes among health care workers exposed to coronavirus disease 2019. JAMA Netw Open. 2020;3(3):e203976. http://doi.org/10.1001/jamanetworkopen.2020.3976.

10. Math SB, Nirmala MC, Moirangthem S, Kumar NC. Disaster management: mental health perspective. Indian J Psychol Med. 2015;37(3):261-71. http://doi.org/10.4103/0253-7176.162915.

11. Tsai KY, Chou P, Chou FHC, Su TTP, Lin SC, Lu MK, et al. Three-year follow-up study of the relationship between posttraumatic stress symptoms and quality of life among earthquake survivors in Yu-chi, Taiwan. J Psychiatr Res. 2007;41(1-2):90-96.

https://doi.org/10.1016/j.jpsychires.2005.10.004.

12. Li S, Wang Y, Xue J, Zhao N, Zhu T. The impact of COVID-19 epidemic declaration on psychological consequences: a study on active Weibo users. Int J Environ Res Public Health. 2020;17(6):2032. https://doi.org/10.3390/ijerph17062032.

13. Wang C, Pan R, Wan X, Tan Y, Xu L, Ho CS, et al. Immediate psychological responses and associated factors during the initial stage of the 2019 coronavirus disease (COVID-19) epidemic among the general population in China. Int J Environ Res Public Health. 2020;17(5):1729. https://doi.org/10.3390/ijerph17051729.

14. Jackson JC, Pandharipande PP, Girard TD, Brummel NE, Thompson JL, Hughes CG, et al. Depression, post-traumatic stress disorder, and functional disability in survivors of critical illness in the BRAINICU study: a longitudinal cohort study. Lancet Respir Med. 2014;2(5):369-379. https://doi.org/10.1016/S2213-2600(14)70051-7.

15. Davydow DS, Desai SV, Needham DM, Bienvenu OJ. Psychiatric morbidity in survivors of the acute respiratory distress syndrome: a systematic review. Psychosom Med. 2008;70(4):512-519. http://doi.org/10.1097/PSY.0b013e31816aa0dd.

16. Dinglas VD, Faraone LN, Needham DM. Understanding patient-important outcomes after critical illness: a synthesis of recent qualitative, empirical, and consensus-related studies. Curr Opin Crit Care. 2018;24(5):401-409. http://doi.org/10.1097/MCC.0000000000000533.

17. Dawson DL, Golijani-Moghaddam N. COVID-19: Psychological flexibility, coping, mental health, and wellbeing in the UK during the pandemic. J Contextual Behav Sci. 2020;17:126-134. https://doi.org/10.1016/j.jcbs.2020.07.010.

18. Guze SB. Why psychiatry is a branch of medicine. New York: Oxford University Press; 1992.

19. Giorgi G, Fiz Perez FS, Castiello D'Antonio A, Mucci N, Ferrero C, Cupelli V, et al. Psychometric properties of the Impact of Event Scale-6 in a sample of victims of bank robbery. Psychol Res Behav Manag. 2015;8:99-104. http://doi.org/10.2147/PRBM.S73901.

20. Hosey MM, Leoutsakos JMS, Li X, Dinglas VD, Bienvenu OJ, Parker AM, et al. Screening for posttraumatic stress disorder in ARDS survivors: Validation of the Impact of Event Scale-6 (IES-6). Crit Care. 2019;23:276. http:/doi.org/10.1186/s13054-019-2553-z. 
21. DiClemente RJ. Knowledge, attitudes and practices related to COVID-19 in the U.S. Inter-University Consortium for Political and Social Research; 2020. https://doi.org/10.3886/E120308V1.

22. Ali SH, Foreman J, Capasso A, Jones AM, Tozan Y, DiClemente RJ. Social media as a recruitment platform for a nationwide online survey of COVID-19 knowledge, beliefs, and practices in the United States: Methodology and feasibility analysis. BMC Med Res Methodol. 2020;20:116. https://doi.org/10.1186/s12874-020-01011-0.

23. Thoresen S, Tambs K, Hussain A, Heir T, Johansen VA, Bisson JI. Brief measure of posttraumatic stress reactions: impact of Event Scale-6. Soc Psychiatry Psychiatr Epidemiol. 2010;45(3):405-412. http://doi.org/10.1007/s00127-009-0073-x.

24. Weiss DS. The Impact of Event Scale: Revised [Internet]. Cross-Cultural Assessment of Psychological Trauma and PTSD. Springer US; p. 219-238. Available from: http://dx.doi.org/10.1007/978-0-38770990-1_10.

25. Czeisler MÉ, Lane RI, Petrosky E, Wiley JF, Christensen A, Njai R, et al. Mental health, substance use, and suicidal ideation during the COVID-19 pandemic-United States, June 24-30, 2020. MMWR Morb Mortal Wkly Rep. 2020;69(32):1049-1057. http://doi.org/10.15585/mmwr.mm6932a1.

26. Williams B, Brown T, Boyle M. Construct validation of the readiness for interprofessional learning scale: a Rasch and factor analysis. J Interprof Care. 2012;26(4):326-332. https://doi.org/10.3109/13561820.2012.671384.

27. Chen W, Zhang G, Tian X, Wang L, Luo J. Rasch analysis of work-family conflict scale among Chinese prison police. Front Psychol. 2021;12:537005. http://doi.org/10.3389/fpsyg.2021.537005.

28. Lambert SD, Pallant JF, Boyes AW, King MT, Britton B, Girgis A. A Rasch analysis of the Hospital Anxiety and Depression Scale (HADS) among cancer survivors. Psychol Assess. 2013;25(2):379-90.

29. Christensen KB, Makransky G, Horton M. Critical values for Yen's Q3: identification of local dependence in the Rasch model using residual correlations. Appl Psychol Meas. 2017;41(3):178-94. http://doi.org/10.1177/0146621616677520.

30. Linacre JM. Optimizing rating scale category effectiveness. J Appl Meas. 2002;3(1):85-106.

31. Wright B. Reasonable mean-square fit values. Rasch Meas Trans. 1994;8:370.

32. Patomella AH, Tham K, Kottorp A. P-drive: Assessment of driving performance after stroke. J Rehabil Med. 2006;38:273-279. http://doi.org/10.1080/16501970600632594.

33. Baghaei P. The effects of the rhetorical organization of texts on the C-test construct: A Rasch modelling study. Melbourne Papers in Language Testing. 2008;13(2): 32-51.

34. Gustafsson J-E. A solution of the conditional estimation problem for long tests in the Rasch model for dichotomous items. Educ Psychol Meas. 1980;40(2):377-385. http://doi.org/10.1177/001316448004000214.

35. Fisher WPJ. Rating scale instrument quality criteria. Rasch Meas Trans. 2007;21(1):1095.

36. Osterlind SJ, Everson HT. Differential item functioning. Newbury Park: Sage Publications, Inc.; 2009. 
37. Reeve BB, Hays RD, Bjorner JB, Cook KF, Crane PK, Teresi JA, et al. Psychometric evaluation and calibration of health-related quality of life item banks: plans for the Patient-Reported Outcomes Measurement Information System (PROMIS). Med Care. 2007;45(5);Suppl 1:S22-31. https://doi.org/10.1097/01.mlr.0000250483.85507.04.

38. Linacre JM. Winsteps Rasch measurement computer program user's guide. Winsteps. Com. 2016.

39. Linacre JM, Wright B. Winsteps help for Rasch analysis. Winsteps. 2012. http://www.winsteps.com. Accessed 15 Sept 2021.

40. Aaronson N, Alonso J, Burnam A, Lohr KN, Patrick DL, Perrin E, et al. Assessing health status and quality-of-life instruments: attributes and review criteria. Qual Life Res. 2002;11(3):193-205.

41. Duncan PW, Bode RK, Min Lai SM, Perera S, Glycine Antagonist in Neuroprotection Americas Investigators. Rasch analysis of a new stroke-specific outcome scale: the Stroke Impact Scale. Arch Phys Med Rehabil. 2003;84(7):950-963. https://doi.org/10.1016/S0003-9993(03)00035-2.

42. American Psychiatric Association. Diagnostic and statistical manual of mental disorders (5th ed.); 2013. https://doi.org/10.1176/appi.books.9780890425596.

43. Karatzias T, Shevlin M, Murphy J, McBride O, Ben-Ezra M, Bentall RP, et al. Posttraumatic stress symptoms and associated comorbidity during the COVID-19 pandemic in Ireland: a population-based study. J Trauma Stress. 2020;33(4):365-370. https://doi.org/10.1002/jts.22565.

44. Murata S, Rezeppa T, Thoma B, Marengo L, Krancevich K, Chiyka E, et al. The psychiatric sequelae of the COVID-19 pandemic in adolescents, adults, and health care workers. Depress Anxiety. 2021;38(2):233-246. https://doi.org/10.1002/da.23120.

45. Hambleton RK, Swaminathan H, Rogers HJ. Fundamentals of item response theory. Vol. 2. Newbury Park: SAGE publications, Inc.; 1991.

46. Armitage R, Nellums LB. The COVID-19 response must be disability inclusive. Lancet Public Health. 2020;5(5):e257. https://doi.org/10.1016/S2468-2667(20)30076-1.

\section{Figures}



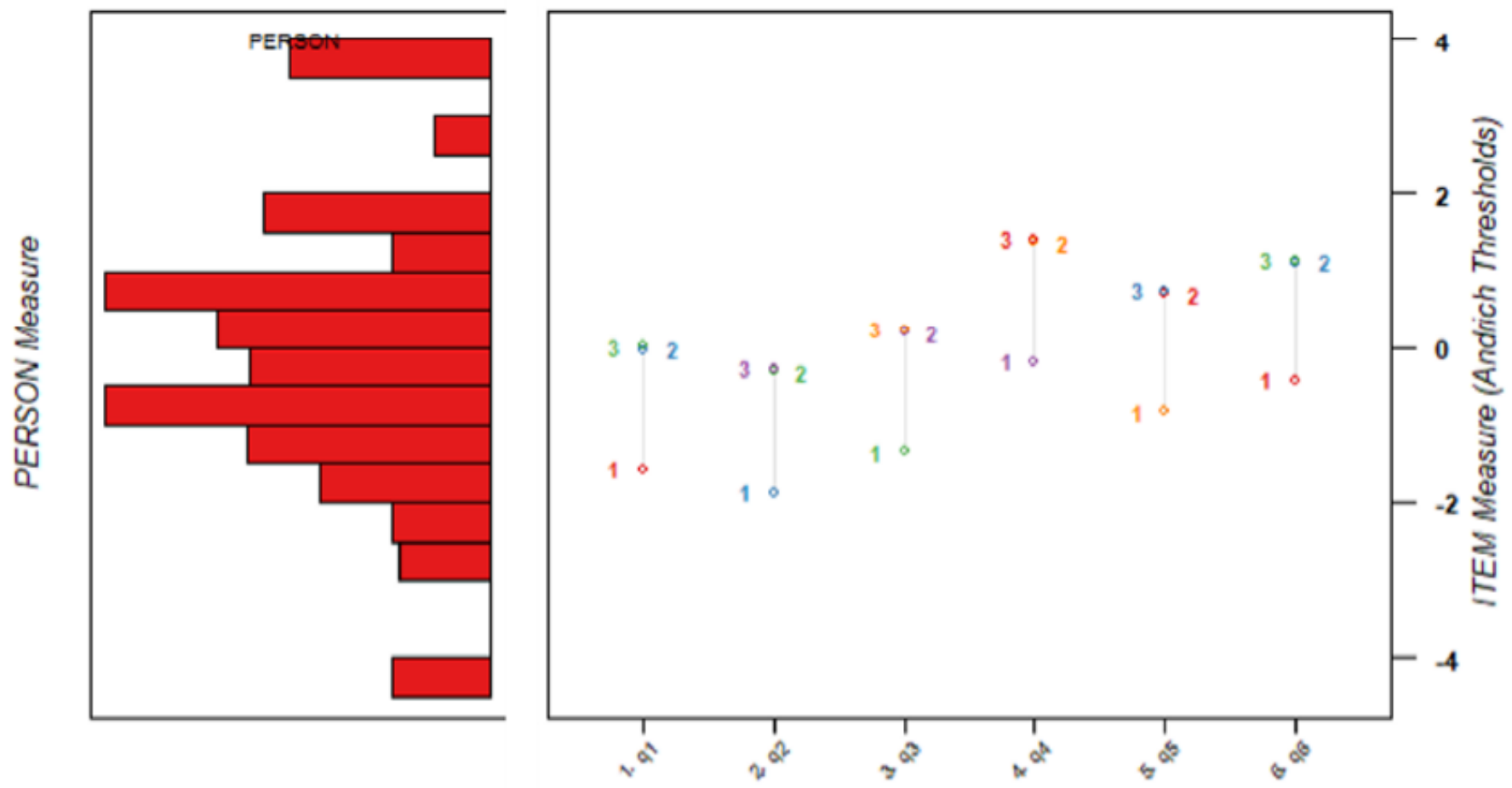

ITEM in Entry order ascending

Figure 1

Wright map

\section{Supplementary Files}

This is a list of supplementary files associated with this preprint. Click to download.

- Additionalfile1.docx 